\title{
Pheochromocytoma and paraganglioma: clinical feature-based disease probability in relation to catecholamine biochemistry and reason for disease suspicion
}

\author{
Aikaterini Geroula1, Timo Deutschbein², Katharina Langton', Jimmy Masjkur', Christina Pamporaki', \\ Mirko Peitzsch ${ }^{3}$, Stephanie Fliedner, Henri J L M Timmers ${ }^{5}$, Stefan R Bornstein ${ }^{4}$, Felix Beuschlein ${ }^{6,7}$, \\ Anthony Stell ${ }^{8}$, Andrzej Januszewicz ${ }^{9}$, Aleksander Prejbisz ${ }^{9}$, Martin Fassnacht ${ }^{2}$, Jacques W M Lenders ${ }^{1,5}$ and \\ Graeme Eisenhofer ${ }^{1,3}$
}

${ }^{1}$ Department of Medicine III, University Hospital Carl Gustav Carus, Technische Universität Dresden, Dresden, Germany, ${ }^{2}$ Division of Endocrinology and Diabetes, Department of Internal Medicine I, University Hospital of Würzburg, Würzburg, Germany, ${ }^{3}$ Institute of Clinical Chemistry and Laboratory Medicine, University Hospital Carl Gustav Carus, Technische Universität Dresden, Dresden, Germany, ${ }^{4}$ First Department of Medicine, University Medical Center Schleswig-Holstein, Lübeck, Germany, ${ }^{5}$ Department of Internal Medicine, Radboud University Medical Center, Nijmegen, The Netherlands, ${ }^{6}$ Medizinische Klinik und Poliklinik IV, Klinikum der Ludwig-Maximilians-Universität München, Munich, Germany, ${ }^{7}$ Department of Endocrinology, Diabetology and Clinical Nutrition, UniviersitätsSpital Zürich, Zürich, Switzerland, ${ }^{8}$ Department of Computing and Information, University of Melbourne, Melbourne, Australia, and ${ }^{9}$ Department of Hypertension, Institute of Cardiology, Warsaw, Poland

Correspondence should be addressed to $\mathrm{G}$ Eisenhofer Email Graeme.Eisenhofer@ uniklinikum-dresden.de

\begin{abstract}
Objective: Hypertension and symptoms of catecholamine excess are features of pheochromocytomas and paragangliomas (PPGLs). This prospective observational cohort study assessed whether differences in presenting features in patients tested for PPGLs might assist establishing likelihood of disease.

Design and methods: Patients were tested for PPGLs because of signs and symptoms, an incidental mass on imaging or routine surveillance due to previous history or hereditary risk. Patients with $(n=245)$ compared to without $(n=1820)$ PPGLs were identified on follow-up. Differences in presenting features were then examined to assess the probability of disease and relationships to catecholamine excess.

Results: Hyperhidrosis, palpitations, pallor, tremor and nausea were $30-90 \%$ more prevalent $(P<0.001)$ among patients with than without PPGLs, whereas headache, flushing and other symptoms showed little or no differences. Although heart rates were higher $(P<0.0001)$ in patients with than without PPGLs, blood pressures were not higher and were positively correlated to $\mathrm{BMI}$, which was lower $(P<0.0001)$ in patients with than without PPGLs. From these differences in clinical features, a score system was established that indicated a 5.8-fold higher probability of PPGLs in patients with high than low scores. Higher scores among patients with PPGLs were associated, independently of tumor size, with higher biochemical indices of catecholamine excess.

Conclusions: This study identifies a complex of five signs and symptoms combined with lower BMI and elevated heart rate as key features in patients with PPGLs. Prevalences of these features, which reflect variable tumoral catecholamine production, may be used to triage patients according to likelihood of disease.
\end{abstract}

(c) 2019 European Society of Endocrinology Printed in Great Britain
European Journal of Endocrinology

(2019) 181, 409-420 


\section{Introduction}

Clinical relevance of pheochromocytomas and paragangliomas (PPGLs) relates to their capacity to secrete catecholamines excessively, thereby producing cardiovascular complications and life-threatening events ( 1 , 2,3 ). Clinical presentation is highly heterogeneous ranging from normotensive and asymptomatic cases to patients with sustained hypertension and others with dramatic swings in blood pressure (BP) accompanied by diverse signs and symptoms during BP highs, even involving hypertensive crises $(4,5,6,7)$. Headaches, hyperhidrosis and palpitations dominate the clinical presentation of PPGLs, particularly during paroxysmal spells $(4,8,9)$. Other features include pallor, panic or anxiety, chest pain, nausea, vomiting, weakness, fatigue, weight loss and constipation (4).

Several factors contribute to the variable clinical manifestations of PPGLs including the type and pattern of catecholamine secretion, sensitivity of impacted adrenoceptors, co-secretion of peptides and tumor size. Patients may also present with a host of comorbid complications (e.g. diabetes, cardiomyopathy, stroke, unexplained shock) that can confuse the clinical picture. Due to these non-specific manifestations, the differential diagnosis is often difficult; consequently, the tumor has been referred to as the 'great mimic' or a 'clinical chameleon' $(4,10)$.

Further complicating diagnosis, there are now changes in how PPGLs are coming to attention. Whereas previously affected patients were mainly diagnosed based on signs and symptoms, today the tumors are increasingly found incidentally as part of imaging studies for purposes unrelated to suspicion of PPGL $(6,11,12)$. Due to recognition of an increasing number of pathogenic mutations and a high rate of disease recurrence, PPGLs are now being found during surveillance screening in patients with a genetic predisposition or past history of disease (11). Such patients can be asymptomatic and normotensive (13).

The changing modes of discovery of today's patients with PPGLs bring to question whether presenting features classically attributed to the tumors remain relevant to likelihood of disease. Furthermore, although the literature is replete with reports on the clinical presentation of patients with PPGLs, there are few comparisons to patients without PPGLs or large-sized studies of patients with and without PPGLs according to today's different modes of disease discovery (14).

The objective of the present study was to identify clinical features of patients tested for PPGLs that might be used to better distinguish those with and without disease and whether such features might also relate to differences in tumoral catecholamine production. This objective was met through a prospective screening study of over 2000 patients tested for PPGLs including 245 patients in whom disease was subsequently confirmed (15). Cardiovascular parameters and other clinical features of tumors were compared to determine those most useful according to whether suspicion of disease was based on periodic surveillance associated with hereditary risk or past history of disease, findings of an incidentaloma or clinical features of catecholamine excess. Examination of presenting features in patients with PPGLs took into account tumor size and biochemical indices of catecholamine excess.

\section{Subjects and methods}

\section{Subjects}

A total of 2291 patients were prospectively recruited into this observational cohort study under a multicenter clinical protocol (Prospective Monoamine-producing Tumor study - PMT) and according to standard-operating procedures (SOPs) available online (https://pmt-study.pressor.org) with further details outlined elsewhere (15) and in the Supplementary methods (see section on supplementary data given at the end of this article). All patients enrolled into the study were recruited according to four study entry criteria: (1) signs and symptoms of presumed catecholamine excess; (2) incidental finding of an abdominal mass requiring further investigation for a PPGL; (3) screening due to previous history of PPGL and (4) hereditary risk of PPGL associated with a mutation of a tumor susceptibility gene.

Registration of patients was facilitated using electronic case report forms (eCRFs) according to a coding system enabling collection and storage of pseudoanonymized patient data as detailed in the Supplementary methods. The PMT study protocol was approved by the University Hospital Dresden Ethics Committee (EC), the Radboud University Medical Center EC, the University Hospital Würzburg EC, the University Medical Center SchleswigHolstein EC, the Klinikum der Ludwig-MaximiliansUniversität München EC and the Institute of Cardiology EC at Warsaw. All subjects provided written informed consent before study inclusion.

\section{Clinical annotations}

Demographic and clinical data (i.e., sex, age, weight, height, systolic and diastolic BP, heart rate, signs, symptoms and antihypertensive medications) were acquired according 
to SOPs at study entry. Patients were evaluated according to a questionnaire to establish occurrence of signs and symptoms over the 30 days before study entry. Signs and symptoms evaluated included headache, hyperhidrosis, palpitations, tremor, pallor, flushing, panic/anxiety, nausea/vomiting, weakness and constipation.

Presence of hypertension was established by systolic BP above or equal to $140 \mathrm{mmHg}$ or diastolic BP above or equal to $90 \mathrm{mmHg}$. Hypertension was also defined in patients with a history of high BP controlled by antihypertensive medications. History of paroxysmal hypertension was assessed from patient notes and interviews.

Mean tumor diameters were calculated from tumor volumes, which were estimated using the formula, $V=\pi / 6$ $(x \times y \times z)$, where $x, y, z$ are the three dimensions. For patients with multifocal primary tumors, tumor volumes were summed. All three dimensions were recorded in 199 $(81.2 \%)$ patients, whereas two diameters in 19 (7.8\%) patients were reported with the third dimension estimated as the average of the two reported dimensions. For four patients $(1.6 \%)$ only one dimension was reported and no information on tumor size was available in 23 (9.4\%) patients, these representing mainly cases of metastatic disease that precluded determination of tumor volume.

\section{Biochemical tests of catecholamine excess}

Biochemical testing at entry of patients into the protocol included mass spectrometric-based measurements of catecholamine metabolites (metanephrine, normetanephrine, methoxytyramine) in their free unconjugated form in plasma and 24-h urine collections, the latter also allowing measurements of catecholamines (epinephrine and norepinephrine). All details including blood and urine collections, reference intervals and designations of tumors as having an epinephrine-producing adrenergic phenotype or a non-adrenergic phenotype are described elsewhere $(15,16,17)$.

\section{Staistical analysis}

Statistical analyses utilized the JMP software package (SAS Institute Inc). The Kruskal-Wallis and the Steel Dwass all-pairs tests were used for non-parametric comparisons of continuous data involving multiple groups. Chisquare and Fisher's exact tests were used to compare frequencies of clinical features and antihypertensive use, with a Bonferroni correction for non-continuous data. Clinical features that showed consistent differences between patients with and without PPGLs were examined in scoring systems to assess probabilities of disease and relationships of scores with tumor size and catecholamine biochemistry. Probabilities of having disease based on the scoring system were calculated according to the basis of disease suspicion (i.e., study inclusion group). Impacts of the presence or absence of PPGLs, study inclusion group, sex, age and BMI on cardiovascular variables and symptom or clinical feature scores were examined by least square multivariate analyses. Relationships of biochemical indices of catecholamine excess with tumor size, study inclusion group and clinical feature scores were similarly examined by multivariate analyses, carried out after normalization of biochemical and tumor size data by logarithmic transformation. Data are displayed as means and $95 \%$ confidence intervals where relevant.

\section{Results}

\section{Patient cohorts: confirmation and exclusion of PPGLS}

After biochemical testing, patients underwent follow-up to confirm or exclude PPGLs; thereafter, the final study population, after exclusions, included 245 patients with PPGLs and 1820 without tumors (Supplementary methods). Due to sample size limitations patients enrolled according to a previous history of PPGLs or hereditary risk were combined into a single group according to the need for periodic surveillance (surveillance group). Among all patients, most $(62.9 \pm 2.1 \%)$ were included based on clinical suspicion due to signs and symptoms compared to smaller proportions included due to an incidentaloma $(23.3 \pm 1.8 \%)$ or as part of surveillance $(13.8 \pm 1.5 \%)$ according to past history of disease or hereditary risk (Table 1). Although there were no significant differences in distributions of sexes, patients with PPGLs were overall younger $(P<0.05)$ than those without PPGLs, this largely reflecting more advanced age in patients of the incidentaloma group who did not have PPGLs.

\section{Cardiovascular variables, body mass index}

BMI was lower $(P<0.0001)$ among patients with than without PPGLs, a difference that was retained $(P<0.01)$ in each of the three study inclusion groups (Table 1). By multivariate analysis, differences in BMI according to presence or absence of PPGLs and study inclusion group were independent of age and sex. Systolic and diastolic BPs were lower $(P<0.001)$ in patients with than without PPGLs, but these differences disappeared after multivariate 
Table 1 Demographics, body mass index, blood pressure and heart rate in patients with and without PPGLs and according to study inclusion group. All data are shown as means with 95\% confidence intervals.

\begin{tabular}{|c|c|c|c|c|}
\hline & All patients & Signs and symptoms & Incidentaloma & Surveillance \\
\hline \multicolumn{5}{|l|}{ Sex (\% male) } \\
\hline No PPGL & $49.5 \pm 2.3 \%(901 / 1820)$ & $51.5 \pm 2.8 \%(621 / 1207)$ & $44.9 \pm 4.9 \%(177 / 394)$ & $47.3 \pm 6.6 \%(103 / 219)$ \\
\hline PPGL & $44.1 \pm 6.2 \%(108 / 245)$ & $43.9 \pm 10.2 \%(40 / 91)$ & $43.2 \pm 10.4 \%(38 / 88)$ & $45.5 \pm 12.0 \%(30 / 66)$ \\
\hline$P$ value & 0.1176 & 0.1920 & 0.8130 & 0.8884 \\
\hline \multicolumn{5}{|l|}{ Age (years) } \\
\hline No PPGL & $52.1 \pm 0.7$ & $50.1 \pm 0.8$ & $58.8 \pm 1.2^{\star \star}$ & $50.9 \pm 2.0^{\dagger}$ \\
\hline PPGL & $49.9 \pm 1.9$ & $48.8 \pm 3.2$ & $53.1 \pm 2.8$ & $47.1 \pm 3.7^{\S}$ \\
\hline$P$ value & 0.0318 & 0.4043 & 0.0004 & 0.0870 \\
\hline \multicolumn{5}{|c|}{ Body mass index $\left(\mathrm{kg} / \mathrm{m}^{2}\right)$} \\
\hline No PPGL & $28.2 \pm 0.3$ & $28.3 \pm 0.3$ & $28.6 \pm 0.6$ & $26.8 \pm 0.7 * *^{\dagger}$ \\
\hline PPGL & $25.0 \pm 0.6$ & $25.3 \pm 0.8$ & $24.9 \pm 1$ & $24 . \overline{8} \pm 1.1$ \\
\hline$P$ value & $<0.0001$ & $<0.0001$ & $<0.0001$ & 0.0063 \\
\hline \multicolumn{5}{|c|}{ Systolic blood pressure (mmHg) } \\
\hline No PPGL & $141.7 \pm 0.9$ & $143.6 \pm 1.2$ & $140.6 \pm 1.9$ & $133.7 \pm 2.6^{* * \dagger}$ \\
\hline PPGL & $135.7 \pm 2.5$ & $139.2 \pm 4.4$ & $136.7 \pm 4.0$ & $129.8 \pm 3.9 *$ \\
\hline$P$ value & $<0.0001$ & 0.0250 & 0.1134 & 0.1628 \\
\hline \multicolumn{5}{|c|}{ Diastolic blood pressure $(\mathrm{mmHg})$} \\
\hline No PPGL & $86.0 \pm 0.6$ & $86.8 \pm 0.6$ & $85.2 \pm 1.1$ & $82.7 \pm 1.5^{\star \star \S}$ \\
\hline PPGL & $82.8 \pm 1.5$ & $82.9 \pm 2.3$ & $83.8 \pm 2.4$ & $81 . \overline{4} \pm 2.8$ \\
\hline$P$ value & 0.0005 & 0.0138 & 0.3878 & 0.2388 \\
\hline \multicolumn{5}{|c|}{ Heart rate (bpm) } \\
\hline No PPGL & $72.1 \pm 0.5$ & $72.3 \pm 0.6$ & $72.7 \pm 1.0$ & $70.3 \pm 1.4^{\S}$ \\
\hline PPGL & $78.8 \pm 1.9$ & $80.7 \pm 2.9$ & $78.4 \pm 3.0$ & $76.9 \pm 2.9$ \\
\hline$P$ value & $<0.0001$ & $<0.0001$ & 0.0002 & $<0.0001$ \\
\hline \multicolumn{5}{|c|}{ Hypertension at study entry } \\
\hline No PPGL & $85.4 \pm 1.6 \%(1540 / 1803)$ & $93.0 \pm 1.5 \%(1111 / 1195)$ & $77.8 \pm 4.1 \%(304 / 391)^{\star *}$ & $57.6 \pm 6.6 \%(125 / 217)^{\star *}$ \\
\hline PPGL & $82.9 \pm 4.7 \%(203 / 245)$ & $94.5 \pm 4.7 \%(86 / 91)$ & $79.6 \pm 8.4 \%(70 / 88)^{\star \star}$ & $71.2 \pm 11 \%(47 / 66)^{\star \star}$ \\
\hline$P$ value & 0.2932 & 0.8293 & 0.7768 & 0.0608 \\
\hline
\end{tabular}

Differences between patients with (PPGL) and without tumors (no PPGL) are shown with $P$ values, whereas difference among the three study inclusion groups are shown according to different symbols: ${ }^{*} P<0.05,{ }^{* *} P<0.001$ different from patients with signs and symptoms; ${ }^{5} P<0.05,{ }^{\dagger} P<0.001$ different from patients with incidentaloma. ${ }^{*} H y p e r t e n s i o n$ at study entry was established according to history of hypertension, antihypertensive use or findings of systolic or diastolic blood pressure above 140 and/or $90 \mathrm{mmHg}$.

correction for age, sex, BMI and study inclusion group (Table 2). In contrast, heart rate was on average $6.5 \mathrm{bpm}$ higher $(P<0.0001)$ in patients with than without PPGLs, a difference that retained significance $(P<0.001)$ in all three patient groups (Table 1) and after multivariate correction for age, sex, BMI and study inclusion group (Table 2).

The prevalence of hypertension at study entry was relatively high among all study inclusion groups, ranging respectively in patients with and without PPGLs from $71 \pm 11 \%$ and $58 \pm 7 \%$ in the surveillance group to $78 \pm 4 \%$ and $80 \pm 8 \%$ in the incidentaloma group and $93 \pm 2 \%$ to $95 \pm 5 \%$ in the signs and symptoms group (Table 1). As expected, patients of the latter group had a higher $(P<0.001)$ prevalence of hypertension than other groups. Although the presence of hypertension in patients with and without PPGLs at study entry did not differ, the prevalence of established hypertension before study entry was higher $(P<0.0001)$ in patients without than with PPGLs (Supplementary Table 1).
Associated with the overall higher prevalence of established hypertension in patients without than with PPGLs, there was a higher $(P<0.0001)$ prescribed use of antihypertensives at study entry for patients without compared to with PPGLs (Supplementary Table 1). As outlined in the Supplementary methods section differences in heart rate and blood pressure in patients with and without PPGLs remained independent of differences in antihypertensive therapy. Reported prevalences of episodic or paroxysmal hypertension did not differ significantly between patients with and without PPGLs ( $35.8 \pm 6 \%$ vs $40.5 \pm 2 \%)$.

\section{Signs and symptoms of catecholamine excess}

Among examined signs and symptoms some such as headaches, flushing and panic/anxiety showed no differences in prevalence between patients with and without PPGLs, including patients tested due to signs and symptoms of 
Table 2 Multivariate analysis of impact of age, sex, body mass index, presence or absence of PPGL and study inclusion group on blood pressure, heart rate and symptom score.

\begin{tabular}{l}
\hline \\
\hline Systolic blood pressure \\
F-ratio \\
$P$ value \\
Impact \\
Diastolic blood pressure \\
F-ratio \\
$P$ value \\
Impact \\
Heart rate \\
F-ratio \\
$P$ value \\
Impact \\
Basic symptom score \\
F-ratio \\
$P$ value \\
Impact
\end{tabular}

\begin{tabular}{c}
\hline Age \\
\hline 51.3 \\
$<0.0001$ \\
+ ve \\
\\
8.0 \\
0.0048 \\
+ ve \\
63.3 \\
$<0.0001$ \\
- ve \\
2.3 \\
0.1295 \\
No
\end{tabular}

\begin{tabular}{c}
\hline Sex \\
\hline 15.1 \\
$<0.0001$ \\
$M>F$ \\
5.0 \\
0.0251 \\
$M>F$ \\
3.1 \\
0.0799 \\
No \\
68.3 \\
$<0.0001$ \\
$F>M$
\end{tabular}

\begin{tabular}{|c|}
\hline BMI \\
\hline $\begin{array}{c}56.1 \\
<0.0001 \\
+ \text { +ve }\end{array}$ \\
\hline $\begin{array}{c}68.4 \\
<0.0001 \\
+ \text { +ve }\end{array}$ \\
\hline $\begin{array}{c}9.7 \\
0.0019 \\
\text { +ve }\end{array}$ \\
\hline $\begin{array}{c}29.0 \\
<0.0001 \\
\text {-ve }\end{array}$ \\
\hline
\end{tabular}

\begin{tabular}{c}
\hline PPGL \\
\hline 0.7 \\
0.4037 \\
No \\
1.7 \\
0.1889 \\
No
\end{tabular}

\begin{tabular}{c}
\hline Inclusion group \\
\hline 23.4 \\
$<0.0001$ \\
$\mathrm{SS}>\mathrm{IN}>\mathrm{SU}$ \\
6.6 \\
0.0015 \\
$\mathrm{SS}>\mathrm{SU}$
\end{tabular}

Impacts for continuous data (i.e., age and BMI) are indicated as +ve or -ve for respective positive and negative relationships or as 'no' where there are no relationships. Impacts for categorical data (i.e., sex, PPGL and study inclusion group) are shown according to the increase of one or two categories above others or as 'no' where there is no impact.

$\mathrm{BMI}$, body mass index; IN, incidentaloma; N, no; PPGL, pheochromocytoma and/or paraganglioma; SS, signs and symptoms; SU, surveillance due to hereditary predisposition or previous history of PPGL; Y, yes.

presumed catecholamine excess (Table 3). In contrast, hyperhidrosis, tremor, pallor and nausea showed 66-102\% higher $(P<0.0001)$ prevalences in patients with than without PPGLs, including consistently higher $(P<0.05)$ prevalences for the first three symptoms for all three study inclusion groups.

Overall the prevalence of palpitations was only $28 \%$ higher $(P=0.0009)$ in patients with than without PPGLs (Table 3). The higher prevalence was independent of prescribed use of beta-adrenergic blockers and differences in heart rate (Supplementary results). Although palpitations had the highest reported prevalence (65\%) in patients with PPGLs of the signs and symptoms group, palpitations were also reported by $44 \%$ of patients without PPGLs indicating non-specificity of this symptom. Muscle weakness and constipation additionally showed 23-60\% higher $(P<0.02)$ prevalences in patients with than without PPGLs, but this did not reach significance for incidentaloma and surveillance groups. Although the classic triad of headaches, hyperhidrosis and palpitations had a relatively high specificity (90\%), only 19\% of patients with PPGLs presented with this triad.

\section{Score system for signs and symptoms and other features of catecholamine excess}

Using the five signs and symptoms that showed significant and consistent differences between patients with and without a PPGL for at least two of the three study inclusion groups, a signs and symptoms score system was established from zero to five according to the sum of points for each sign (pallor) and symptom (hyperhidrosis, palpitations, tremor and nausea). According to receiver-operating characteristic curves the combination of this symptom score with HR and BMI yielded higher $(P<0.0001)$ areas under the curve than any feature alone (Supplementary Fig. 1).

Multivariate analysis accounting for age, sex, BMI, presence or absence of PPGLs and study inclusion group established, as expected, higher $(P<0.0001)$ scores in patients with than without PPGLs and higher $(P<0.0001)$ scores in patients tested for PPGLs in the signs and symptoms group than other groups (Table 2). Sex and BMI, but not age, were other factors associated with differences $(P<0.0001)$ in signs and symptoms score, with female sex showing a particularly strong impact toward higher scores.

Among the 669 patients with a BMI less than $25 \mathrm{~kg} /$ $\mathrm{m}^{2}, 20.0 \%$ had PPGLs compared to $12.0 \%$ for all patients, indicating a $67 \%$ higher probability of tumors. In contrast, among the 584 patients who were categorized as obese (i.e., BMI $>30 \mathrm{~kg} / \mathrm{m}^{2}$ ) only $4.8 \%$ had PPGLs indicating a $60 \%$ lower probability of PPGLs. Additionally, as selected from comparisons of interquartiles, $26.2 \%$ of patients with PPGLs had a heart rate of $85 \mathrm{bpm}$ or more compared to $12.2 \%$ of patients without PPGLs. Because of these 
Table 3 Signs and symptoms of catecholamine excess in patients with and without PPGLs and according to study inclusion group. All data are shown as means with 95\% confidence intervals.

\begin{tabular}{|c|c|c|c|c|}
\hline & All patients & Signs and symptoms & Incidentaloma & Surveillance \\
\hline \multicolumn{5}{|l|}{ Headaches } \\
\hline No PPGL & $39.0 \pm 2.2 \%(692 / 1774)$ & $44.9 \pm 2.8 \%(525 / 1170)$ & $29.2 \pm 4.5 \%(113 / 387)^{\star \star}$ & $24.9 \pm 5.7 \%(54 / 217)^{\star \star}$ \\
\hline PPGL & $38.3 \pm 6.1 \%(93 / 243)$ & $46.1 \pm 10.3 \%(41 / 89)$ & $38.6 \pm 10.1 \%(34 / 88)^{\star *}$ & $27.3 \pm 10.7 \%(18 / 66)^{\star}$ \\
\hline$P$ value & 0.6130 & 0.4558 & 0.0562 & 0.4044 \\
\hline \multicolumn{5}{|c|}{ Hyperhydrosis } \\
\hline No PPGL & $27.6 \pm 2.1 \%(489 / 1774)$ & $29.7 \pm 2.6 \%(347 / 1170)$ & $25.1 \pm 4.3 \%(97 / 387)$ & $20.7 \pm 5.3 \%(45 / 217)^{*}$ \\
\hline PPGL & $45.7 \pm 6.2 \%(111 / 243)$ & $55.1 \pm 10.3 \%(49 / 89)$ & $46.7 \pm 10.4 \%(41 / 88)$ & $31.8 \pm 11.2 \%(21 / 66)^{*}$ \\
\hline$P$ value & $<0.0001$ & $<0.0001$ & $<0.0001$ & 0.0471 \\
\hline \multicolumn{5}{|l|}{ Palpitations } \\
\hline No PPGL & $36.0 \pm 2.2 \%(639 / 1774)$ & $43.9 \pm 2.8 \%(514 / 1170)$ & $21.7 \pm 4.11 \%(84 / 387)^{\star *}$ & $18.9 \pm 5.2 \% 41 / 217)^{\star \star}$ \\
\hline PPGL & $46.1 \pm 6.2 \%(112 / 243)$ & $65.2 \pm 9.9 \%(58 / 89)$ & $45.5 \pm 10.4 \%(40 / 88)^{*}$ & $21.2 \pm 9.8 \%(14 / 66)^{* \star \dagger}$ \\
\hline$P$ value & 0.0016 & $<0.0001$ & $<0.0001$ & 0.3985 \\
\hline \multicolumn{5}{|l|}{ Tremor } \\
\hline No PPGL & $14.6 \pm 1.6 \%(259 / 1774)$ & $18.4 \pm 2.2 \%(215 / 1170)$ & $8.3 \pm 2.7 \%(32 / 387 * *$ & $5.5 \pm 3.03 \%(12 / 217)^{\star *}$ \\
\hline PPGL & $25.1 \pm 5.4 \%(61 / 243)$ & $32.6 \pm 9.7 \% 29 / 89)$ & $20.5 \pm 8.4 \%(20 / 88)$ & $18.2 \pm 9.3 \%(12 / 66)$ \\
\hline$P$ value & $<0.0001$ & 0.0015 & 0.0065 & 0.0127 \\
\hline \multicolumn{5}{|l|}{ Pallor } \\
\hline No PPGL & $13.0 \pm 1.5 \%(230 / 1774)$ & $15.0 \pm 2.0 \%(176 / 1170)$ & $9.8 \pm 2.9 \%(38 / 387)^{*}$ & $7.4 \pm 3.4 \%(16 / 217)^{*}$ \\
\hline PPGL & $26.2 \pm 5.5 \%(63 / 243)$ & $37.1 \pm 10.0 \%(33 / 89)$ & $20.5 \pm 8.4 \%(18 / 88)^{*}$ & $18.2 \pm 9.3 \%(12 / 66)^{*}$ \\
\hline$P$ value & $<0.0001$ & $<0.0001$ & 0.0065 & 0.0127 \\
\hline \multicolumn{5}{|l|}{ Flushing } \\
\hline No PPGL & $22.9 \pm 1.9 \%(406 / 1774)$ & $26.6 \pm 2.5 \%(311 / 1170)$ & $16.5 \pm 3.7 \%(64 / 387)^{\star *}$ & $14.3 \pm 4.6 \%(31 / 217)^{\star *}$ \\
\hline PPGL & $19.8 \pm 5.0 \%(48 / 243)$ & $21.4 \pm 8.5 \%(19 / 89)$ & $22.7 \pm 8.7 \% 20 / 88)$ & $13.6 \pm 8.2 \%(9 / 66)^{\star}$ \\
\hline$P$ value & 0.8817 & 0.8881 & 0.1130 & 0.6218 \\
\hline \multicolumn{5}{|c|}{ Panic/anxiety } \\
\hline No PPGL & $24.6 \pm 2.0 \%(437 / 1774)$ & $31.5 \pm 2.6 \%(368 / 1170)$ & $14.0 \pm 3.4 \% 54 / 387)^{\star \star}$ & $6.9 \pm 3.3 \%(15 / 2176)^{* \star \dagger}$ \\
\hline PPGL & $24.7 \pm 5.4 \%(60 / 243)$ & $37.1 \pm 10.0 \%(33 / 89)$ & $19.3 \pm 8.2 \%(17 / 88)^{*}$ & $15.2 \pm 8.6 \%(10 / 66)^{\star}$ \\
\hline$P$ value & 0.5198 & 0.1633 & 0.2451 & 0.0394 \\
\hline \multicolumn{5}{|c|}{ Nausea/vomiting } \\
\hline No PPGL & $11.4 \pm 1.4 \%(202 / 1774)$ & $11.7 \pm 1.8 \%(137 / 1170)$ & $11.1 \pm 3.1 \%(43 / 387)$ & $10.1 \pm 4.01 \%(22 / 217)$ \\
\hline PPGL & $21.4 \pm 5.1 \%(52 / 243)$ & $25.8 \pm 9.0 \%(23 / 89)$ & $18.2 \pm 8.0 \%(16 / 88)$ & $19.7 \pm 9.6 \%(13 / 66)$ \\
\hline$P$ value & $<0.0001$ & 0.0004 & 0.0550 & 0.036 \\
\hline \multicolumn{5}{|l|}{ Weakness } \\
\hline No PPGL & $34.6 \pm 2.2 \%(614 / 1774)$ & $36.1 \pm 2.7 \%(422 / 1170)$ & $31.8 \pm 4.6 \%(123 / 387)$ & $31.8 \pm 6.2 \%(69 / 217)$ \\
\hline PPGL & $42.4 \pm 6.2 \%(103 / 243)$ & $50.6 \pm 10.3 \%(45 / 89)$ & $34.1 \pm 9.9 \%(30 / 88)^{*}$ & $42.4 \pm 11.9 \%(28 / 66)$ \\
\hline$P$ value & 0.0112 & 0.0049 & 0.3820 & 0.0753 \\
\hline \multicolumn{5}{|l|}{ Constipation } \\
\hline No PPGL & $8.5 \pm 1.3 \%(151 / 1774)$ & $8.6 \pm 1.6 \%(100 / 1170)$ & $9.0 \pm 2.8 \%(35 / 387)$ & $7.4 \pm 3.4 \%(16 / 217)$ \\
\hline PPGL & $13 . \overline{6} \pm 4.3 \%(33 / 243)$ & $16.9 \pm 7.7 \%(15 / 89)$ & $10.2 \pm 6.32 \%(9 / 88)$ & $13.6 \pm 8.2 \%(9 / 66)$ \\
\hline$P$ value & 0.0093 & 0.0116 & 0.4301 & 0.0963 \\
\hline \multicolumn{5}{|c|}{ Classic triad (headaches, hyperhidrosis, palpitations) } \\
\hline No PPGL & $9.8 \pm 1.3 \%(173 / 1774)$ & $11.3 \pm 1.8 \%(132 / 1170)$ & $7.5 \pm 2.6 \%(29 / 387)$ & $5.5 \pm 3.03 \%(12 / 217)^{*}$ \\
\hline PPGL & $19.3 \pm 4.9 \%(47 / 243)$ & $23.6 \pm 8.8 \%(21 / 89)$ & $23.9 \pm 8.9 \%(21 / 88)$ & $7.6 \pm 6.3 \%(5 / 66)^{\star \dagger}$ \\
\hline$P$ value & $<0.0001$ & 0.0013 & $<0.0001$ & 0.3602 \\
\hline
\end{tabular}

Differences between patients with tumors (PPGL) and without tumors (no PPGL) are shown with $P$ values, whereas differences between the three study inclusion groups are shown according to different symbols: ${ }^{*} P<0.05,{ }^{*} * P<0.001$ different from patients with signs and symptoms; ${ }^{\dagger} P<0.05$ different from patients with incidentaloma.

differences, the basic signs and symptoms score was adapted with inclusion of BMI and heart rate. For this the scoring system was amended with a negative point for obese patients and an extra point for patients with a BMI less than $25 \mathrm{~kg} / \mathrm{m}^{2}$. A single point was added for heart rates equal to or above $85 \mathrm{bpm}$, providing a maximal total score of 7 points. Low clinical feature scores of -1 to 0 were present in $35.0 \%$ of the study population, but only $4.2 \%$ of low scoring patients had PPGLs. In contrast, high scores of 3 or more were present in $21.2 \%$ of patients among whom $24.5 \%$ had PPGLs.

As shown in Table 4, the likelihood of having a PPGL among patients with a high clinical feature score was 5.8fold higher than for patients with a low score, but this 
Table 4 Probabilities (\%) for disease (PPGL) in all patients and according to low, medium and high clinical feature scores or according to study inclusion groups.

\begin{tabular}{|c|c|c|c|c|}
\hline & All patients & Signs and symptoms & Incidentaloma & Surveillance \\
\hline \multicolumn{5}{|l|}{ All patients } \\
\hline PPGL (yes/no) & $241 / 1721$ & $90 / 1147$ & $86 / 363$ & $65 / 211$ \\
\hline Probability & $12.3 \pm 1.5 \%$ & $7.3 \pm 1.4 \%$ & $19.2 \pm 3.6 \%$ & $23.6 \pm 5.0 \%$ \\
\hline \multicolumn{5}{|c|}{ Low clinical feature score $(-1$ or 0$)$} \\
\hline PPGL (yes/no) & 29/661 & $6 / 408$ & $10 / 165$ & $13 / 88$ \\
\hline Probability & $4.2 \pm 1.5 \%$ & $1.5 \pm 1.2 \%$ & $5.7 \pm 3.4 \%$ & $12.9 \pm 6.5 \%$ \\
\hline \multicolumn{5}{|c|}{ Medium clinical feature score ( 1 or 2 ) } \\
\hline PPGL (yes/no) & $110 / 746$ & $34 / 499$ & $42 / / 152$ & $34 / 95$ \\
\hline Probability & $12.9 \pm 2.2 \%$ & $6.4 \pm 2.1 \%$ & $21.7 \pm 5.8 \%$ & $26.4 \pm 7.6 \%$ \\
\hline \multicolumn{5}{|c|}{ High clinical feature score $(\geq 3)$} \\
\hline PPGL (yes/no) & $102 / 314$ & $50 / 240$ & $34 / 46$ & $18 / 28$ \\
\hline Probability & $24.5 \pm 4.1 \%$ & $17.2 \pm 4.3 \%$ & $42.5 \pm 10.8 \%$ & $39.1 \pm 14.1 \%$ \\
\hline
\end{tabular}

Probabilities are shown as means with 95\% confidence intervals.

varied from a 3.0-fold higher likelihood in the surveillance group to 7.5- and 11.5-fold higher respective likelihoods in incidentaloma and signs and symptoms groups.

\section{Relationships of catecholamine biochemistry with clinical feature scores and tumor size}

Plasma and urinary free metanephrines as well as urinary catecholamines showed expected differences among patients with and without PPGLs according to reasons for biochemical tests (Supplementary Table 2). Patients with PPGLs showed differences in urinary catecholamines as well as urinary and plasma-free metanephrines according to designation in the three clinical feature score groups (Fig. 1). Specifically all three biochemical indices of catecholamine excess were higher $(P<0.02)$ in patients with high clinical feature scores than those with low and medium scores. Plasma and urinary free metanephrines were also higher $(P<0.05)$ in patients with medium than low scores.

In contrast to the differences in catecholamine biochemistry, mean tumor diameter did not differ among patients with low, medium or high scores (Fig. 1). Tumor diameter nevertheless showed strong positive relationships with plasma metanephrines and urinary free metanephrines, but weaker relationships with urinary catecholamines (Fig. 2). According to those relationships examined according to clinical feature score group (Fig. 2, panels A, B and C), study inclusion group (Fig. 2, panels D and E), multivariate analyses (Supplementary Table 3) or examination of indices of catecholamine excess relative to tumor diameter (Supplementary Fig. 2), it was clear that indices of catecholamine excess varied independently of tumor size according to both clinical feature score and inclusion groups. Specifically, patients with high clinical feature scores had larger increases $(P<0.005)$ in indices of catecholamine excess relative to tumor size than patients with low sores. Furthermore, patients in surveillance and incidentaloma groups had smaller increases $(P<0.05)$ in indices of catecholamine excess relative to tumor size than patients in the signs and symptoms group.

Patients with adrenergic epinephrine-producing tumors were older $(P<0.0001)$ had higher $(P=0.0269)$ basic symptom scores as well as higher $(P<0.05)$ prevalences of tremor, pallor and panic/anxiety compared to patients with PPGLs that did not produce significant amounts of epinephrine (Table 5).

\section{Discussion}

This study provides unique data from comparisons of clinical features among a large group of patients screened for PPGLs and from this identifies key discriminating features for a scoring system to assess the likelihood of the disease. Links of discriminating features to differences in tumoral catecholamine production were identified, thereby clarifying why some patients do not present with the same features as others even when tumors are similarly sized. Unlike previous efforts the present investigation considered underlying reasons for clinical suspicion of disease and covered patients with and without PPGLs identified from the same cohort prospectively tested for disease. In this way the data provide novel and valuable information for physicians about how to assess the likelihood of a PPGL in relation to clinical suspicion based on signs and symptoms or the increasingly more common indications of an incidentaloma or surveillance based on genetic risk or past history of the tumors. 

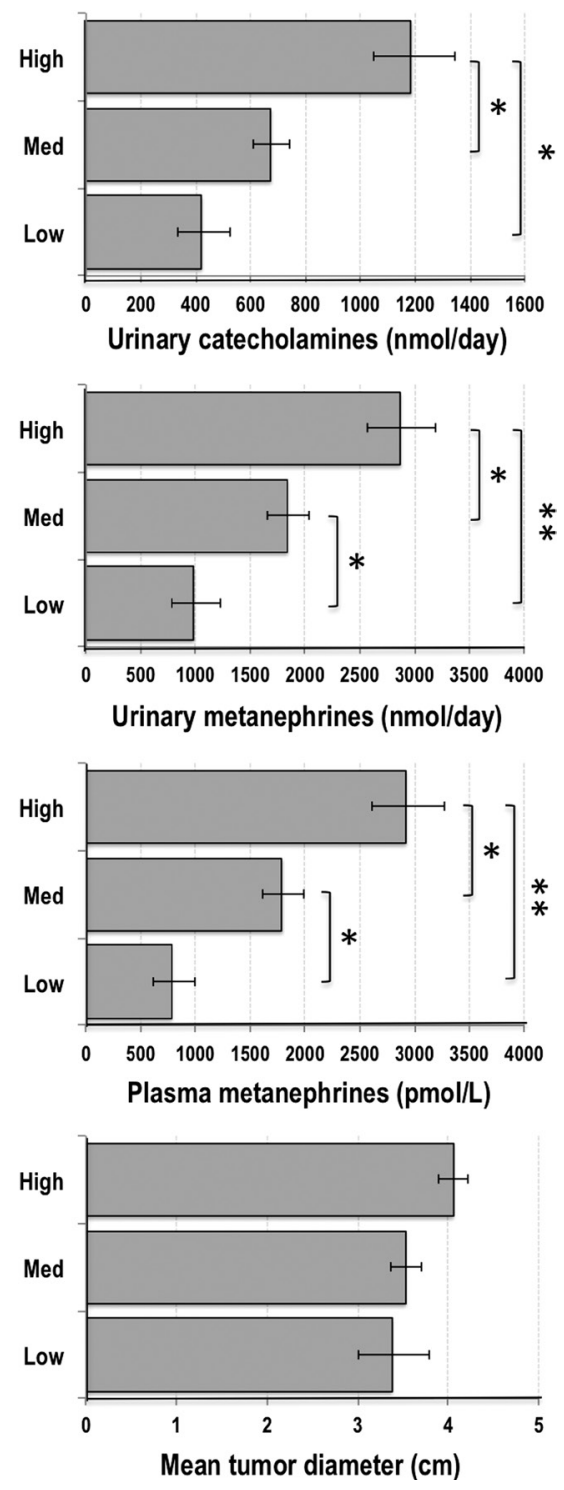

Figure 1

Differences in urinary catecholamines, urinary free metanephrines, plasma metanephrines and mean tumor diameter according to low, medium (Med) and high presentation scores among patients with PPGLs. Presentation scores were based on a point for the presence of each of the top five signs or symptoms that showed significant differences between patients with and without PPGLs, an additional point each for a heart rate of 85 or more or a body mass index $<25 \mathrm{~kg} / \mathrm{m}^{2}$ with subtraction of one point for a body mass index $>30 \mathrm{~kg} / \mathrm{m}^{2}$. Patients with scores of -1 or 0 were assigned to the low score group, those with scores of 1 or 2 to the medium score group and those with scores above 3 to the high score group. Data are restricted to the $90.6 \%$ of patients with PPGLs in whom measurements of mean tumor diameter were possible. ${ }^{*} P<0.05, * * P<0.001$
As clarified in a systematic review of the literature paired down to 36 studies reporting on the clinical presentation of patients with PPGLs (14), most reports do not provide comparative data from patients without tumors. Among the few with comparative data, most involved highly selected or limited numbers of patients (18, 19, 20). In 1981 Plouin and colleagues established that the triad of headache, palpitations and hyperhidrosis was present in 10 of the 11 patients (91\%) with PPGLs
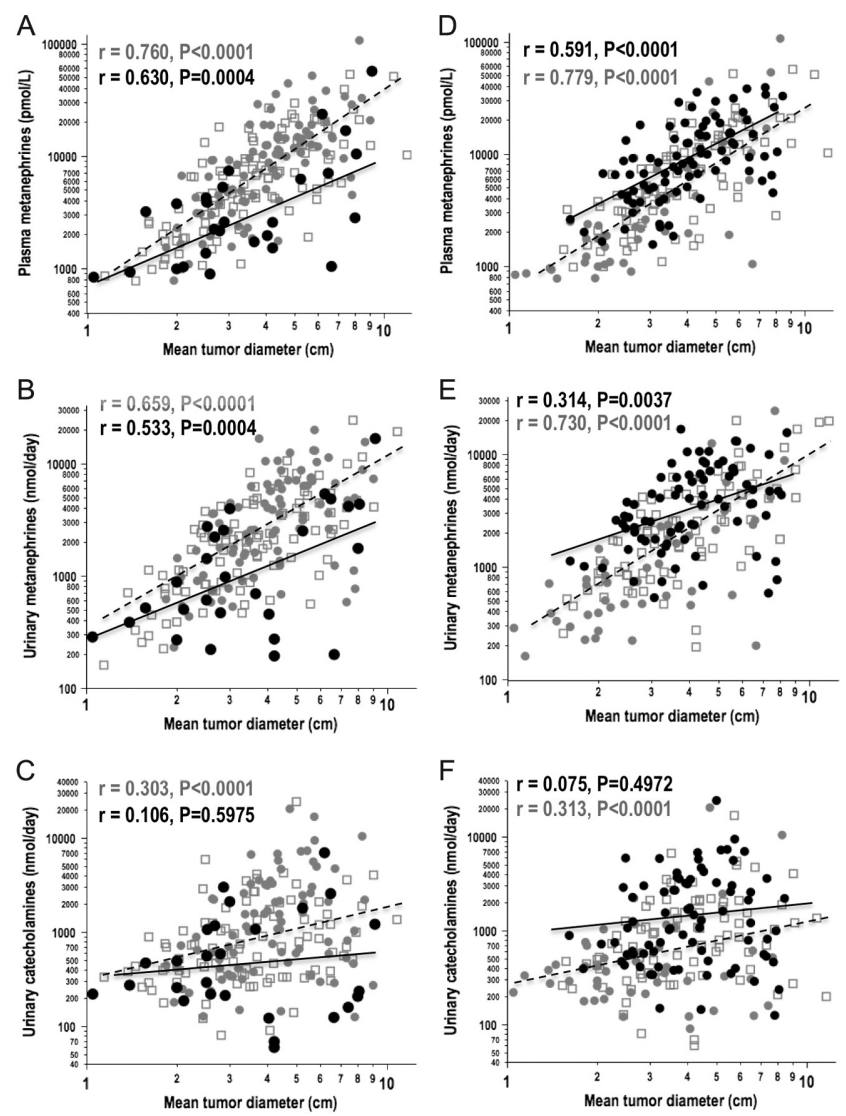

Figure 2

Relationships of mean tumor diameter with plasma free metanephrines (panels $A$ and $D$ ), urinary free metanephrines (panels B and E) and urinary catecholamines (panels C and F) in patients with PPGLs. Relationships for panels $A, B$ and $C$ are separately shown for patients with high $(\odot)$ and medium $(\square)$ presentation score groups compared to the low score group (๑). Relationships for panels D, E and F are separately shown for patients tested due to an incidentaloma ( $\square$ ) or undergoing routine screening due to previous history or genetic risk (O) compared to patients tested due to signs and symptoms of catecholamine excess ( ). Data are restricted to the $90.6 \%$ of patients with PPGLs in whom measurements of mean tumor diameter were possible. 
compared to $6.5 \%$ of the 2585 patients with hypertension suggesting utility of the triad for assessing likelihood of disease (8). A subsequent report in 1984 from Black and Bursten (9) outlined a scoring system that combined signs and symptoms with biochemical test results from 60 patients with PPGLs, another 25 in whom PPGLs were excluded and 410 hypertensives. As in the study of Plouin et al., headache, hyperhidrosis and palpitations were the dominant presenting features in patients tested for PPGLs. Nevertheless, although present at higher prevalences in patients with than without PPGLs, the triad also exhibited a relative high frequency among those without PPGLs illustrating its non-specific nature. Subsequent reports, although limited to patients with PPGLs, have indicated relatively lower proportions of patients presenting with the 'classic triad' $(21,22$, 23). This is in keeping with the findings here that the symptom triad was present in less than $25 \%$ of patients with PPGLs and at prevalences on average only two-fold higher than in patients without PPGLs.

The aforementioned shortcomings in published reports mean that although there exists a constellation of established clinical features for catecholamine-producing tumors, it remains unclear how these translate into meaningful information for triaging patients according to likelihood of disease. The present report addresses this need by defining relative prevalences of clinical features in patients with and without PPGLs. In most cases the findings are in accord with accepted views, in others they are not. For example many signs and symptoms of apparent catecholamine excess were as expected more prevalent in patients with than without PPGLs; however, others such as headache were not. While this and the relatively small differences in prevalences between the two groups support the non-specific nature of these features, it was nevertheless a surprise that office measurements of BP and presence hypertension were not helpful in assessing likelihood of PPGLs. Another study has additionally indicated lower BP in patients with PPGLs than essential hypertensives (24). These findings are consistent with the conclusions of a meta-analysis indicating that hypertension is not important compared to other features to assess probability of PPGLs and that normotension might in fact be a more important consideration (14).

While the above findings and conclusions appear to fly in the face of accepted dogma, there is a need to consider that at least for the group evaluated because of signs and symptoms, these patients would be expected to have an a priori high prevalence of hypertension independent of the presence of PPGLs. Also important are the findings derived from multivariate analyses showing that the lower BPs in patients with than without PPGLs lost significance after correction for age, sex and specially the lower BMI in patients with than without PPGLs. Significant impacts of catecholamine-producing tumors on body weight, adiposity and energy metabolism are well documented $(25,26,27)$, with differences in BMI reflecting differences in catecholamines (28). Here we show that among patients tested for PPGLs, those with a BMI lower than $25 \mathrm{~kg} / \mathrm{m}^{2}$ have a higher prevalence compared to a lower prevalence of PPGLs in obese patients. Thus, both heart rate and $\mathrm{BMI}$, but not office $\mathrm{BP}$, can provide information about likelihood of PPGLs in patients suspected with or at risk of PPGLs.

On the basis of the above findings, a score system was devised that integrated information from selected signs and symptoms with BMI and heart rate. Hyperhidrosis, palpitations, tremor, pallor and nausea provided the five components of the signs and symptoms complex selected based on consistency of differences between groups with and without PPGLs. With the resulting clinical feature score system, relative probabilities of disease according to low-to-high scores can be combined with information on disease prevalence to refine estimates of disease probability ahead of triaging patients and biochemical testing. As detailed in the Supplementary discussion such information can be useful for determining probabilities of disease after biochemical testing. Thus, for patients with high pre-test prevalences of PPGLs, such as those with incidentalomas, the presence of a high versus a low clinical feature score in combination with even small to mildly increased biochemical test results could define a difference between more rapidly advancing to surgery as opposed to further follow-up confirmatory biochemical testing or functional imaging. For other patients with low pre-test prevalences of PPGLs, low score lack of any relevant clinical features may minimize need for biochemical testing, particularly compared to patients with high clinical feature scores who are more likely to harbor a PPGL.

Most clinical manifestations of PPGLs result from hemodynamic and metabolic actions of catecholamines secreted by the tumors. Accordingly and as expected, symptomatic patients with high clinical feature scores showed higher urinary catecholamines than those with lower scores. They also showed higher plasma and urinary free metanephrines, which reflects catecholamine vesicular store size and continuous leakage of catecholamines from those stores into the cytoplasm followed by local metabolism (29). Interestingly patients with low clinical 
Table 5 Adrenergic phenotype* of PPGLs and disease presentation. All variables are shown as means with confidence intervals.

\begin{tabular}{l}
\hline Sex (\% males) \\
Age (years) \\
BMI $\left(\mathrm{kg} / \mathrm{m}^{2}\right)$ \\
SBP $(\mathrm{mmHg})$ \\
DBP $(\mathrm{mmHg})$ \\
HR (bpm) \\
Headache \\
Hyperhidrosis \\
Palpitations \\
Tremor \\
Pallor \\
Flushing \\
Panic/anxiety \\
Nausea/vomiting \\
Weakness \\
Constipation \\
Symptom score
\end{tabular}

\begin{tabular}{c}
\hline Adrenergic \\
\hline $38.3 \%(49 / 128)$ \\
$54.1 \pm 2.7$ \\
$25.5 \pm 0.8$ \\
$136.7 \pm 3.7$ \\
$82.6 \pm 2.2$ \\
$78.5 \pm 2.6$ \\
$33.9 \pm 8.2 \%(43 / 127)$ \\
$45.7 \pm 8.7 \%(58 / 127)$ \\
$51.2 \pm 8.7 \%(65 / 127)$ \\
$30.7 \pm 8.0 \%(39 / 127)$ \\
$34.4 \pm 6.9 \%(44 / 127)$ \\
$19.7 \pm 8.3 \%(25 / 127)$ \\
$32.3 \pm 8.1 \%(41 / 127)$ \\
$26.0 \pm 7.6 \%(33 / 127)$ \\
$41.0 \pm 8.6 \%(52 / 127)$ \\
$14.2 \pm 6.1 \%(18 / 127)$ \\
$1.86 \pm 0.28$
\end{tabular}

\begin{tabular}{c}
\hline Not adrenergic \\
\hline $50.4 \%(59 / 117)$ \\
$45.3 \pm 2.4$ \\
$24.4 \pm 0.8$ \\
$134.5 \pm 3.1$ \\
$82.9 \pm 2.2$ \\
$79.2 \pm 3.0$ \\
$43.1 \pm 9.0 \%(50 / 116)$ \\
$45.7 \pm 9.1 \%(53 / 116)$ \\
$40.5 \pm 8.9 \%(47 / 116)$ \\
$19.0 \pm 7.1 \%(22 / 116)$ \\
$16.4 \pm 6.7 \%(19 / 116)$ \\
$19.8 \pm 7.3 \%(23 / 116)$ \\
$16.4 \pm 6.7 \%(19 / 116)$ \\
$16.4 \pm 6.7 \%(19 / 116)$ \\
$44.0 \pm 9.0 \%(51 / 116)$ \\
$12.9 \pm 6.1 \%(15 / 116)$ \\
$1.38 \pm 0.22$
\end{tabular}

\begin{tabular}{r}
\hline P value \\
\hline 0.0712 \\
$<0.0001$ \\
0.0417 \\
0.8371 \\
0.9383 \\
0.9728 \\
0.1481 \\
1.0000 \\
0.1220 \\
0.0388 \\
0.0013 \\
1.0000 \\
0.0046 \\
0.0849 \\
0.6970 \\
0.8523 \\
0.0269
\end{tabular}

*The adrenergic phenotype of PPGLs was determined from plasma concentrations of metanephrine relative to the other two metabolites as outlined in the Subjects and methods section and serves to designate PPGLs that produce significant amounts of epinephrine compared to other tumors that do not.

feature scores were characterized by tumors that produced catecholamines at lower rates relative to tumor diameter than patients with higher scores. Thus, more highly concentrated stores of catecholamines in some tumors and associated higher rates of catecholamine secretion may contribute to why some patients with similarly sized PPGLs are diagnosed on the basis of signs and symptoms while others present as incidentalomas. This conclusion is consistent with other reports of lower indices of catecholamine production and secretion in patients with PPGLs presenting as incidentalomas than for other reasons $(5,12)$.

Other differences in disease presentation have been suggested to relate to the relative abundances at which PPGLs secrete epinephrine versus norepinephrine (30, 31, 32). In keeping with this concept, patients with epinephrine-producing adrenergic tumors were characterized by an overall higher symptom score and in particular more tremor, pallor and panic/anxiety than patients with PPGLs that did not produce epinephrine. Other findings consistent with those of previous reports include the higher ages of disease presentation for patients with adrenergic than other PPGLs (33), higher reported prevalence of signs and symptoms in females than males (34), and the expectedly higher prevalences of hypertension and biochemical indices of catecholamine excess in patients with PPGLs suspected due to signs and symptoms compared to other indications $(11,23)$.

While the prospective nature of patient recruitment, large sample size and inclusion of patients with and without PPGLs represent study strengths there are limitations, such as difficulties in establishing paroxysmal spells (Supplementary discussion). Despite those limitations, this study does provide unique data to better facilitate clinical interpretation of presenting features in patients with suspected PPGLs. While the limited value of office BP measurements for determining likelihood of PPGLs was a surprising finding that departs from established dogma, this may in part reflect changes in how the tumors are nowadays detected along with a rising prevalence of obesity and associated hypertension in the general population.

\section{Supplementary data}

This is linked to the online version of the paper at https://doi.org/10.1530/ EJE-19-0159.

\section{Declaration of interest}

Martin Fassnacht is on the editorial board of EJE. Martin Fassnacht was not involved in the review or editorial process for this paper, on which he is listed as an author. The other authors have nothing to disclose.

\section{Funding}

This work was supported by the Deutsche Forshungsgemeinschaft (DFG, German Research Foundation Project number: 314061271-CRC/TRR 205/1).

\section{Acknowledgements}

The authors thank Denise Kaden for technical assistance, Stephanie Zopp and Yvonne Möhres for assistance with clinical studies as well as Drs Christina Berr and Finn Strasding for help with patient management. 


\section{References}

1 Plouin PF, Duclos JM, Soppelsa F, Boublil G \& Chatellier G. Factors associated with perioperative morbidity and mortality in patients with pheochromocytoma: analysis of 165 operations at a single center. Journal of Clinical Endocrinology and Metabolism 200186 1480-1486. (https://doi.org/10.1210/jcem.86.4.7392)

2 Prejbisz A, Lenders JW, Eisenhofer G \& Januszewicz A. Cardiovascular manifestations of phaeochromocytoma. Journal of Hypertension 2011 29 2049-2060. (https://doi.org/10.1097/HJH.0b013e32834a4ce9)

3 Riester A, Weismann D, Quinkler M, Lichtenauer UD, Sommerey S, Halbritter R, Penning R, Spitzweg C, Schopohl J, Beuschlein F et al. Life-threatening events in patients with pheochromocytoma. European Journal of Endocrinology 2015173 757-764. (https://doi. org/10.1530/EJE-15-0483)

4 Manger WM and Gifford RW. Clinical and Experimental Pheochromocytoma, 2nd ed. Cambridge, MA: Blackwell Publishing Science, 1996.

5 Noshiro T, Shimizu K, Watanabe T, Akama H, Shibukawa S, Miura W, Ito $\mathrm{S} \&$ Miura $\mathrm{Y}$. Changes in clinical features and long-term prognosis in patients with pheochromocytoma. American Journal of Hypertension 200013 35-43. (https://doi.org/10.1016/s0895-7061(99)00139-9)

6 Kopetschke R, Slisko M, Kilisli A, Tuschy U, Wallaschofski H, Fassnacht M, Ventz M, Beuschlein F, Reincke M, Reisch N et al. Frequent incidental discovery of phaeochromocytoma: data from a German cohort of 201 phaeochromocytoma. European Journal of Endocrinology 2009161 355-361. (https://doi.org/10.1530/EJE-09-0384)

7 Zuber SM, Kantorovich V \& Pacak K. Hypertension in pheochromocytoma: characteristics and treatment. Endocrinology and Metabolism Clinics of North America 201140 295-311, vii. (https:// doi.org/10.1016/j.ecl.2011.02.002)

8 Plouin PF, Degoulet P, Tugaye A, Ducrocq MB \& Menard J. Screening for phaeochromocytoma : in which hypertensive patients? A semiological study of 2585 patients, including 11 with phaeochromocytoma (author's transl). Nouvelle Presse Medicale 1981 10 869-872.

9 Black HR \& Bursten SL. A clinical scoring system for detection of patients with pheochromocytomas. Yale Journal of Biology and Medicine 198457 259-272.

10 Eisenhofer G, Lenders JWM, Manger WM \& Pacak K. Pheochromocytoma: a clinical chameleon. In Autonomic Failure: $A$ Textbook of Clinical Disorders of the Autonomic Nervous System, 5th ed., pp 770-785. Eds CJ Mathias \& R Bannister. Oxford: Oxford University Press, 2013.

11 Gruber LM, Hartman RP, Thompson GB, McKenzie TJ, Lyden ML, Dy BM, Young WF \& Bancos I. Pheochromocytoma characteristics and behavior differ depending on method of discovery. Journal of Clinical Endocrinology and Metabolism 2019104 1386-1393. (https:// doi.org/10.1210/jc.2018-01707)

12 Wachtel H, Cerullo I, Bartlett EK, Roses RE, Cohen DL, Kelz RR, Karakousis GC \& Fraker DL. Clinicopathologic characteristics of incidentally identified pheochromocytoma. Annals of Surgical Oncology 201522 132-138. (https://doi.org/10.1245/s10434-0143933-x)

13 Mannelli M, Lenders JW, Pacak K, Parenti G \& Eisenhofer G. Subclinical phaeochromocytoma. Best Practice and Research: Clinical Endocrinology and Metabolism 201226 507-515. (https://doi. org/10.1016/j.beem.2011.10.008)

14 Soltani A, Pourian M \& Davani BM. Does this patient have pheochromocytoma? A systematic review of clinical signs and symptoms. Journal of Diabetes and Metabolic Disorders 2015156. (https://doi.org/10.1186/s40200-016-0226-x)

15 Eisenhofer G, Prejbisz A, Peitzsch M, Pamporaki C, Masjkur J, Rogowski-Lehmann N, Langton K, Tsourdi E, Pęczkowska M, Fliedner $\mathrm{S}$ et al. Biochemical diagnosis of chromaffin cell tumors in patients at high and low risk of disease: plasma versus urinary free or deconjugated O-methylated catecholamine metabolites. Clinical Chemistry 201864 1646-1656. (https://doi.org/10.1373/ clinchem.2018.291369)

16 Eisenhofer G, Peitzsch M, Kaden D, Langton K, Mangelis A, Pamporaki C, Masjkur J, Geroula A, Kurlbaum M, Deutschbein T et al. Reference intervals for LC-MS/MS measurements of plasma free, urinary free and urinary acid-hydrolyzed deconjugated normetanephrine, metanephrine and methoxytyramine. Clinica Chimica Acta: International Journal of Clinical Chemistry 2019490 46-54. (https://doi.org/10.1016/j.cca.2018.12.019)

17 Eisenhofer G, Klink B, Richter S, Lenders JW \& Robledo M. Metabologenomics of phaeochromocytoma and paraganglioma: an integrated approach for personalised biochemical and genetic testing. Clinical Biochemist: Reviews 201738 69-100.

18 Plouin PF, Chatellier G, Rougeot MA, Duclos JM, Pagny JY, Corvol P \& Ménard J. Recent developments in pheochromocytoma diagnosis and imaging. Advances in Nephrology from the Necker Hospital 198817 $275-286$.

19 Stein PP \& Black HR. A simplified diagnostic approach to pheochromocytoma. A review of the literature and report of one institution's experience. Medicine 199170 46-66. (https://doi. org/10.1097/00005792-199101000-00004)

20 Yu R, Nissen NN, Chopra P, Dhall D, Phillips E \& Wei M. Diagnosis and treatment of pheochromocytoma in an academic hospital from 1997 to 2007. American Journal of Medicine 2009122 85-95. (https:// doi.org/10.1016/j.amjmed.2008.08.021)

21 Mannelli M, Ianni L, Cilotti A \& Conti A. Pheochromocytoma in Italy: a multicentric retrospective study. European Journal of Endocrinology 1999141 619-624. (https://doi.org/10.1530/ eje.0.1410619)

22 Cohen DL, Fraker D \& Townsend RR. Lack of symptoms in patients with histologic evidence of pheochromocytoma: a diagnostic challenge. Annals of the New York Academy of Sciences 20061073 47-51. (https://doi.org/10.1196/annals.1353.005)

23 van Duinen N, Steenvoorden D, Bonsing BA, Vuyk J, Vriends AH, Jansen JC, Romijn JA \& Corssmit EP. Pheochromocytomas detected by biochemical screening in predisposed subjects are associated with lower prevalence of clinical and biochemical manifestations and smaller tumors than pheochromocytomas detected by signs and symptoms. European Journal of Endocrinology 2010163 121-127. (https://doi.org/10.1530/EJE-10-0114)

24 Stolk RF, Bakx C, Mulder J, Timmers HJ \& Lenders JW. Is the excess cardiovascular morbidity in pheochromocytoma related to blood pressure or to catecholamines? Journal of Clinical Endocrinology and Metabolism 201398 1100-1106. (https://doi.org/10.1210/ jc.2012-3669)

25 Petrak O, Haluzikova D, Kavalkova P, Strauch B, Rosa J, Holaj R, Brabcová Vránková A, Michalsky D, Haluzík M, Zelinka T et al. Changes in energy metabolism in pheochromocytoma. Journal of Clinical Endocrinology and Metabolism 201398 1651-1658. (https:// doi.org/10.1210/jc.2012-3625)

26 Spyroglou A, Adolf C, Hahner S, Quinkler M, Ladurner R, Reincke M $\&$ Beuschlein F. Changes in body mass index in pheochromocytoma patients following adrenalectomy. Hormone and Metabolic Research 201749 208-213. (https://doi.org/10.1055/s-0042-124189)

27 Okamura T, Nakajima Y, Satoh T, Hashimoto K, Sapkota S, Yamada E, Okada S, Fukuda J, Higuchi T, Tsushima Y et al. Changes in visceral and subcutaneous fat mass in patients with pheochromocytoma. Metabolism: Clinical and Experimental 201564 706-712. (https://doi. org/10.1016/j.metabol.2015.03.004)

28 An Y, Reimann M, Masjkur J, Langton K, Peitzsch M, Deutschbein T, Fassnacht M, Rogowski-Lehmann N, Beuschlein F, Fliedner S et al. Adrenomedullary function, obesity and permissive influences of catecholamines on body mass in patients with chromaffin cell tumours. International Journal of Obesity 201943 263-275. (https:// doi.org/10.1038/s41366-018-0054-9) 
29 Eisenhofer G, Goldstein DS, Kopin IJ \& Crout JR. Pheochromocytoma: rediscovery as a catecholamine-metabolizing tumor. Endocrine Pathology 200314 193-212. (https://doi. org/10.1007/s12022-003-0012-4)

30 Lance JW \& Hinterberger H. Symptoms of pheochromocytoma, with particular reference to headache, correlated with catecholamine production. Archives of Neurology 197633 281-288. (https://doi. org/10.1001/archneur.1976.00500040065011)

31 Ito Y, Fujimoto Y \& Obara T. The role of epinephrine, norepinephrine, and dopamine in blood pressure disturbances in patients with pheochromocytoma. World Journal of Surgery 199216 759-763; discussion 763-754. (https://doi.org/10.1007/BF02067379)

32 Eisenhofer G, Walther MM, Huynh TT, Li ST, Bornstein SR, Vortmeyer A, Mannelli M, Goldstein DS, Linehan WM, Lenders JW et al. Pheochromocytomas in von Hippel-Lindau syndrome and multiple endocrine neoplasia type 2 display distinct biochemical and clinical phenotypes. Journal of Clinical Endocrinology and Metabolism 200186 1999-2008. (https://doi.org/10.1210/ jcem.86.5.7496)

33 Eisenhofer G, Timmers HJ, Lenders JW, Bornstein SR, Tiebel O, Mannelli M, King KS, Vocke CD, Linehan WM, Bratslavsky G et al. Age at diagnosis of pheochromocytoma differs according to catecholamine phenotype and tumor location. Journal of Clinical Endocrinology and Metabolism 201196 375-384. (https://doi. org/10.1210/jc.2010-1588)

34 Lai EW, Perera SM, Havekes B, Timmers HJ, Brouwers FM, McElroy B, Adams KT, Ohta S, Wesley RA, Eisenhofer G et al. Gender-related differences in the clinical presentation of malignant and benign pheochromocytoma. Endocrine 200834 96-100. (https://doi. org/10.1007/s12020-008-9108-4)

Received 4 March 2019

Revised version received 17 June 2019

Accepted 31 July 2019 